ROCZNIKI HUMANISTYCZNE

Tom LXIX, zeszyt 5 - 2021

DOI: http://doi.org/10.18290/rh21695-11

MONIKA GRZESZCZAK

\title{
DER DEMOKRATIEBEGRIFF UND SEINE PROFILIERUNG IM DEUTSCHEN UND POLNISCHEN. EINE KONTRASTIVE ANALYSE
}

\begin{abstract}
A bstract. Der Beitrag setzt sich zum Ziel, den Demokratiebegriff im Deutschen und Polnischen kontrastiv zu behandeln sowie die Arten seiner Konzeptualisierung im deutschen und polnischen öffentlichen Diskurs der Gegenwart aufzuzeigen. Die methodologische Grundlage bilden die Prämissen der Lubliner kognitiven Ethnolinguistik. Als Materialbasis dienen dabei drei Arten von Quellen, und zwar: Wörterbücher der deutschen und polnischen Sprache, experimentelle Befragungen sowie Pressetexte. Auf deren Grundlage wird ein Bündel von Grundeigenschaften (den sog. „Basiseigenschaften“) im Verständnis von ,Demokratie“ in beiden behandelten Sprachen festgelegt, das allen Diskursen gemeinsam ist. Im Anschluss daran wird auf die Merkmale von ,Demokratie“ hingewiesen, die in den ideologisch und weltanschaulich differenzierten Diskursen ans Licht kommen. Die Profilierung des Begriffs wird anhand des Textmaterials aus der deutschen und polnischen Presse mit folgender Ausrichtung skizzenhaft dargestellt: liberalkonservativ und linksliberal.
\end{abstract}

Schlüsselwörter: Demokratie; Diskurs; Profilierung; Wörterbuch; Umfrage.

\section{EINLEITUNG}

Demokratie ist ein weit verbreiteter Ausdruck, der einerseits zum gemeinsamen europäischen sprachlich-kulturellen Erbe (dem sog. Paneuropäismus ${ }^{1}$ ) gehört, andererseits dessen Grenzen überschreitet und im internationalen öffentlichen Raum als Internationalismus angesehen wird. Dafür sprechen Eigenschaften dieser lexikalisch-semantischen Einheit wie u.a. die Zugehörigkeit zu den „internationalen lexikalischen Ressourcen“, die Entstehung

Dr. Monika GrzeszczaK - Katholische Johannes-Paul-II.-Universität Lublin, Institut für Linguistik, Lehrstuhl für Germanistische Linguistik; Korrespondenzadresse: Al. Racławickie 14, 20 950 Lublin; e-mail: monika.grzeszczak@kul.pl; ORCID: https://orcid.org/0000-0002-4549-3721.

${ }^{1}$ Ausführlicher zu diesem Thema siehe: Bartmiński, „Pasywne i aktywne paneuropeizmy“. 
auf Grundlage griechischer Wörter (über die lateinische Sprache) oder ihre Verbreitung in mehreren nicht verwandten bzw. nur sehr entfernt verwandten Sprachen (vgl. Waszakowa 25).

Das Ziel des vorliegenden Beitrags besteht in einer Vergleichsanalyse zum Verständnis des Demokratiebegriffs in den Sprachen Deutsch und Polnisch. Darüber hinaus sollen die Arten seiner Konzeptualisierung im deutschen und im polnischen öffentlichen Diskurs der Gegenwart aufgezeigt werden. Die methodologische Grundlage bilden die Konzepte der kognitiven Ethnolinguistik, die auf Grundlage der Analysen der Ethnolinguistischen Schule von Lublin ausgearbeitet, definiert und angewendet wurden ${ }^{2}$. Den Gegenstand dieses Gebiets linguistischer Studien bildet „die Sprache in all ihren Varietäten, darunter auch der allgemeinen, in ihrer Beziehung zu Kultur, Mensch und Gesellschaft" (Niebrzegowska-Bartmińska 80) ${ }^{3}$. Vom ethnolinguistischen „Standpunkt her kann man sagen, Wörter sind wie Kristalle, die in ihrem Inneren das Bild der Welt brechen und deren ausgewählte Aspekte bündeln; Wörter verbinden sich durch ein Beziehungsgeflecht mit anderen Wörtern, Gegenständen, Menschen, mit Fakten der eigenen Geschichte und der Geschichte der Sprachgemeinschaft. Das Studium der lexikalischen Ressourcen der Sprache und der Bedeutung der Wörter eröffnet eine Perspektive auf die gesamte Kultur.“ (Bartmiński, „Czym zajmuje się etnolingwistyka“"18).

Zunächst wird eine Gruppe grundlegender Definitionseigenschaften zum Verständnis von ,Demokratie“ herausgearbeitet (die sog. Gruppe der „Basiseigenschaften“), die in den Lexika und im „Allgemeinwissen“ festgelegt sind. Anschließend wird versucht, anhand des gesammelten Textmaterials die Frage zu beantworten, welche Eigenschaftsgruppen der ,Demokratie ' auf Grundlage der spezialisierten, hinsichtlich der Ideologie und Weltanschauung differenzierten Diskurse zugeschrieben werden. „Diese Diskurse [...] vollziehen unterschiedlich ausgerichtete semantische Modifikationen der Grundbedeutung“ (Bartmiński, „Wartości i ich profile medialne“ 34). Unter den Lubliner Ethnolinguisten positioniert sich der Begriff des Diskurses, der zwar an sich ,unscharf" und mehrdeutig ist - im Unterschied zum Text als einem „sprachlichen Erzeugnis eines Kommunikationsaktes (Akte)“ - auf Ebene der Sprachpraxis. Er bezieht sich dabei nicht einfach auf „,die Sprache in ihrem Gebrauch', sondern umfasst zusätzlich den gesamten situa-

\footnotetext{
${ }^{2}$ Dazu siehe u.a. die Bände JOS und ACE.

${ }^{3}$ Alle Zitate aus dem Polnischen sowie alle Wörter und Wortverbindungen dieser Sprache wurden von der Autorin übersetzt.
} 
tiven Kontext, das kommunikative System in seiner gesamten Komplexität, das der Interaktion und der Umsetzung der vorgegebenen Ziele dient. Er ist eine prozessuale und kontextbezogene Konzeptualisierung ,des Kommunikationsereignisses', dessen gegenständliche, ,erzeugende' Konzeptualisierung der Text ist." (Bartmiński und Niebrzegowska-Bartmińska 32). Dieser Teil der Arbeit stützt sich auf das Konzept der Profilierung der Begriffe (siehe Band PP und PJT). Dabei wird unter Profilierung ,eine subjektive (d.h. über ein Subjekt verfügende) sprachlich-begriffliche Operation [verstanden], die darin besteht, das Bild eines Objekts spezifisch zu gestalten, indem es unter bestimmten Aspekten aufgefasst wird [...] im Rahmen eines bestimmten Wissenstyps, den Anforderungen einer bestimmten Sichtweise entsprechend." (Bartmiński und Niebrzegowska 212).

Als Materialgrundlage dienen drei Typen von Daten: Systemdaten, Umfragedaten und Textdaten. Bei der Datenerhebung wurden Wörterbücher der deutschen und der polnischen Sprache, etymologische Wörterbücher, Synonymwörterbücher, Wörterbücher der sinnverwandten Wörter und Fremdwörterbücher verwendet. Die Arbeit nimmt außerdem - nacheinander - Bezug auf die Ergebnisse von Umfragen, die in Lublin insgesamt drei Mal durchgeführt wurden (als ASA ${ }^{4}$ 1990, ASA 2000 und ASA 2010 bezeichnet) sowie auf die Ergebnisse empirischer Studien, die unter Studierenden deutscher Universitäten und Hochschulen im Jahre 2019 durchgeführt wurden (als FB 2019 gekennzeichnet). Die Textdaten umfassen Pressetexte aus Tages- und Wochenzeitungen, in denen verschiedene Weltanschauungen und ideologische Standpunkte vertreten werden ${ }^{6}$.

\footnotetext{
${ }^{4}$ ASA $=$, ,ankieta słownika aksjologicznego“ [„,Umfrage zum axiologischen Wörterbuch“]. Initiiert und etabliert wurde die Umfrage von den Ethnolinguisten aus Lublin.

${ }^{5} \mathrm{FB}=$ Fragebogen. Eine umfassende Beschreibung der Umfrage einschließlich einer Liste der deutschen Universitäten und Hochschulen findet sich in dem Beitrag „Obraz ,demokracji“ (,Demokratie‘) w języku niemieckim w świetle badań ankietowych“ [,Das Bild der ,Demokratie“ im Deutschen im Lichte experimenteller Untersuchungen (Umfragen)"]. Artikel im Druck.

${ }^{6} \mathrm{Im}$ vorliegenden Beitrag werden nur ausgewählte Dimensionen des Demokratiebegriffs in verschiedenen öffentlichen Diskursen in Deutschland und in Polen vorgestellt. Das gesamte Bild des Demokratiebegriffs unter Berücksichtigung der umfangreichen Profilierung wurde in der Buchpublikation mit dem Titel „Pojęcie ,demokracji“ i jego profilowanie w polskim i niemieckim dyskursie publicznym (1989-2009)“ [,Der Demokratiebegriff und seine Profilierung im polnischen und deutschen öffentlichen Diskurs (1989-2009)“] erfasst. Siehe Literaturverzeichnis.
} 


\section{DEMOKRATIE IN WÖRTERBÜCHERN DER DEUTSCHEN SPRACHE}

Das Lexem Demokratie wurde im 16. Jh. in der lateinischen Form democratia ,Herrschaft des Volks“ (EW Klu), dann als democraty [1592] (DW Paul) erfasst. Ältere Wörterbücher der deutschen Sprache sowie die Lexika des 20. Jhs. verzeichnen zwischen einem und vier Bedeutungen dieses Lexems ${ }^{7}$. Etwas verallgemeinert lässt sich feststellen, dass sich in den meisten lexikografischen Publikationen folgende Auffassungen von Demokratie wiederfinden: a) ,eine Herrschaftsform, in der die Macht vom Volk ausgeht'; b) ,eine Form des Staatssystems, in der die oberste Macht beim Volk/allen Bürgern liegt'; c), ein Staat mit demokratischem System'; d), ein politisches Prinzip, bei dem das Volk durch freie Wahlen an der Ausübung der Herrschaft im Staat beteiligt ist'; e), ein Prinzip des gesellschaftlichen Lebens, das auf freien und gleichberechtigten Entscheidungen und Mitentscheidungen beruht ${ }^{\circ}$.

Wie die Wörterbuchdaten zeigen, ist die am stärksten konsolidierte Eigenschaft der Demokratie, die zugleich das grundlegende und konstitutive Bedeutungselement dieses Lexems in der deutschen Sprache darstellt, die Assoziation mit einer Herrschaftsform, bei der die Grundlage und Quelle jeglicher Macht das Volk ist, ursprünglich verstanden als die unteren sozialen Schichten (Plebs, gemeines Volk), später als die Gesamtheit der Bürger. Dieses Attribut der Demokratie bestätigt u.a. das „Allgemeine verdeutschende und erklärende Fremdwörterbuch [...]“" von Johann Ch.A. Heyse (AFW Hey), das als Erstes einige auf Grundlage der deutschen Sprache gebildete synonymische Kompositionen wie etwa die Volksherrschaft, Volksregierung, das Volksreich oder Bürgerreich verzeichnet. Dass es sich bei der Eigenschaft ,Form der Herrschaft, beruhend auf der Macht des Volkes, das im Grunde genommen die Mehrheit der Gesellschaft darstellt‘ um eine Kern- bzw. eine primäre Eigenschaft in der Konzeptualisierung der Demokratie handelt, belegt auch die Etymologie des Wortes. Den etymologischen Wörterbüchern zufolge ${ }^{9}$ wurde das Wort Demokratie aus dem Griechischen über das Lateinische in die deutsche Sprache

\footnotetext{
${ }^{7}$ Das Lexem Demokratie ist nicht im „Wörterbuch der deutschen Sprache“ der Gebrüder Grimm verzeichnet, das allgemein als eines der wichtigsten und umfangreichsten Lexika gilt.

${ }^{8}$ Siehe FW San; FWB; GKW Adel; AFW Hey; WDG KlapStein; DW Wah; DudDUW; DudFW.

${ }^{9}$ Siehe DudEHW, DEW Köb, EWD Pfei, REW.
} 


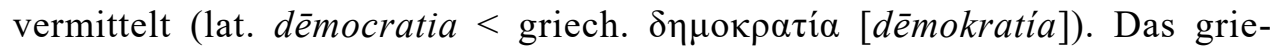
chische Wort besteht dabei aus zwei Teilen, und zwar aus $\delta \tilde{\eta} \mu$ os [démos]

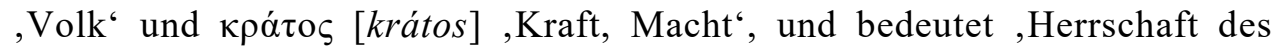
Volkes, der Vielen, der Mehrheit der Volksversammlung' (DFW).

Das Lexem Demokratie wird meistens - im Hinblick auf den sozialen Aspekt - mit Freiheit assoziiert. Diese Eigenschaft bestätigen einige in den Wörterbüchern verzeichnete Kollokationen wie z.B. freie Demokratie, freiheitliche Demokratie (DudSW), außerdem Bedeutungsexplikationen verwandter Wörter wie: Demokrat ,ein Freibürger, Freiheitsfreund' (AFW Hey); demokratisch ,freiheitlich“ (DudSW)/ ,freibürgerlich, freiländisch“ (AFW Hey); demokratisieren ,freibürgerliche Gesinnungen hegen und äußern“ (AFW Hey); Demokratismus, der Freibürgersinn' (AFW Hey). Demokratie wird außerdem mit Freiheit und Menschenrechten gleichgesetzt, bspw.: „Fahnenwörter sind Wörter mit einer positiven Wertung wie Freiheit, Demokratie, Menschenrechte“ (GW Mül).

Bedeutungsexplikationen von Derivaten sowie - seltener - von Wortverbindungen, in denen sie vorkommen, belegen, dass zur Semantik der Demokratie neben der Freiheit auch die Gleichheit der Bürger gehört (bspw. Demokrat, Anhänger einer Staatsform, in der die Staatsbürger gleich und frei sind"; DW Mack), bzw. Eigenschaften wie gleichberechtigte Mitwirkung ${ }^{10}$ sowie Berücksichtigung/Respektierung des Willens der Mehrheit der Bürger bzw. der parlamentarischen Mehrheit ${ }^{11}$.

\section{DEMOKRACJA IN WÖRTERBÜCHERN DER POLNISCHEN SPRACHE}

Das Lexem demokracja, das auch in der Form democratia [1564], demokracyja geschrieben wurde, tauchte im Polnischen im 16. Jh. auf. Dies bestätigt das „Słownik polszczyzny XVI wieku“ (SPXVI), in dem demokracja in seiner etymologischen Grundbedeutung definiert wird, nämlich als ,Herrschaftsform, die auf der Macht des Volkes beruht.' Ältere und zeitgenössische Wörterbücher der polnischen Sprache verzeichnen von einer bis $\mathrm{zu}$ vier Bedeutungen dieses Lexems. Etwas verallgemeinert lässt sich feststellen, dass sich in den meisten lexikografischen Publikationen folgende

\footnotetext{
10 „demokratisch (unter gleichberechtigter Mitwirkung aller Beteiligten) gefasste Beschlüsse“ (HW Kemp).

${ }^{11}$ demokratisch, in einer Weise, die den Willen der Mehrheit berücksichtigt‘ (DudBW).
} 
Auffassungen von demokracja wiederfinden: a), eine Herrschaftsform, in der die Staatsgewalt durch das Volk ausgeübt wird'; b) , eine Form des Staatssystems, in der die Staatsgewalt beim Volk/bei der Mehrheit der Bürger (Mehrheit der Gesellschaft) oder bei deren Vertretern liegt ${ }^{6}$; c), ein Staat mit einem solchen System '; d) ,Fraktion, Partei, (manchmal soziale Gruppe), die eine demokratische Herrschaft unterstützt ${ }^{12}$.

Den Wörterbuchdaten zufolge ist die am stärksten konsolidierte Eigenschaft von demokracja, die man zugleich zu den konstitutiven, in der sog. „Kernzone“ verankerten Bedeutungen des Wortes zählen kann, die Assoziation mit der Herrschaftsform, die auf der Macht des Volkes beruht, das ursprünglich als die untere Schicht, später als, die Gesamtheit der Menschen jeglichen Standes, Geschlechts und Alters, die gemeinsam versammelt sind oder in einem Land wohnen' aufgefasst wurde (SSP Kras). Die Meinung, bei der Demokratie handle es sich um ein Herrschaftssystem, das von unteren sozialen Schichten (gemeinem Volk, Pöbel) ausgeübt wird, konsolidierte sich in synonymen Formen wie z.B. gminowładztwo, gminorzadztwo (SW). Seit der zweiten Hälfte des 19. Jhs. (siehe SWil) wird als Synonym zu demokracja im Sinne der Herrschaftsform und später des politischen Systems vornehmlich der Ausdruck ludowtadztwo bzw. - als Periphrase - władza $l u d u$ verwendet. Darauf, dass es sich bei der Eigenschaft ,Herrschaftsform, basierend auf der Macht des Volkes' um eine primäre Grundeigenschaft in der Konzeptualisierung der Demokratie handelt, deutet auch die Etymologie des Wortes hin. Dem „Nowy słownik etymologiczny języka polskiego“ von Krystyna Długosz-Kurczabowa (NSEJP Dług-Kur) zufolge ist demokracja ein Wort, das aus dem Griechischen (griech. demokratia) über das Lateinische (lat. democratía) entliehen wurde und aus zwei Teilen besteht: démos - ,Volk' und krátos - ,Kraft, Macht, Gewalt; politische Macht' und wörtlich die ,Macht des Volkes' bedeutet.

In die Semantik von demokracja fügt sich die axiologische Triade Freiheit, Gleichheit, Gerechtigkeit ein. Die Eigenschaft der Freiheit bestätigen einige synonymische Ausdrücke des analysierten Lexems wie z.B.: wolnomyślicielstwo, wolnomyślność, liberalizm (SSiA). Auf Gleichheit als semantische Komponente der Demokratie deuten wiederum Synonyme des von demokracja abgeleiteten Wortes demokratyczny hin wie równy, gleich“ (für alle) und egalitarny ,egalitär' (SSP Kurz). Unter Gleichheit wird dabei gleiches Recht für alle verstanden (demokratisch bedeutet bei SWB LPT-H

\footnotetext{
${ }^{12}$ Siehe SPXVI; SL; SWil; SIJP; SJP Dor; SJP Szym; PSWP Zgół; ISJP Bań; USJP Dub; SWJP Dun; SJP DSSW; ISJP Sob.
} 
dasselbe wie gleichberechtigt) bzw. als gleiches Recht auf Beteiligung an Entscheidungen, die alle betreffen: „Etwas, was ,demokratisch“ ist, basiert auf der Überzeugung, dass alle über gleiche Rechte verfügen und gleichsam an Entscheidungen über die Allgemeinheit beteiligt werden sollen." (ISJP Bań). Herangezogen wird dabei u.a. die politische Gleichheit, die implizit das Stimmrecht, das Recht auf Gewaltenkontrolle und das Recht auf Versammlung zwecks Meinungsäußerung enthält. Demokratie wird - seltener - mit Gerechtigkeit gleichgesetzt, insbesondere mit sozialer Gerechtigkeit (SSiA).

Dass die Demokratie in der politischen Dimension mit der Macht der Mehrheit der Bürger, in der sozialen Dimension hingegen mit Rechten und bürgerlichen Freiheiten gleichgesetzt wird, bestätigt u.a. die Aussage, die man im Definitionsteil des Stichworts demokrata, einer von demokracja derivierten lexikalischen Einheit, lesen kann: ,Befürworter der Demokratie, eine Person mit demokratischen Überzeugungen, die den Willen der Mehrheit der Bürger als Quelle der Macht anerkennt und die sich für bürgerliche Rechte und Freiheiten ausspricht‘ (PSWP Zgół).

\section{DER DEMOKRATIEBEGRIFF IM LICHTE DER UMFRAGEDATEN}

Wenn man die oben nur skizzenhaft vorgestellten Wörterbuchdaten mit den Umfragedaten vergleicht, so bestätigt die Umfrage die Verwurzelung der politischen und sozialen Eigenschaften in der Konzeptualisierung der ,Demokratie', außerdem - was zweifellos als Vorzug dieser Methode gesehen werden kann - erlaubt sie die Erstellung einer Rangliste dieser Eigenschaften sowie die Benennung von neuen Eigenschaften, die von den Wörterbuchautoren bisher nicht einbezogen wurden.

Das zur Analyse des polnischen Demokratiebegriffs herangezogene Umfragematerial wurde von einer Forschungsgruppe zusammengetragen, die unter der Leitung von Jerzy Bartmiński das Thema „Axiologischer Wortschatz im Gegenwartspolnisch“ bearbeitete ${ }^{13}$. Den Studierenden der Lubliner Hochschulen (ca. 100 Personen, die Hälfte davon Studenten/Studentinnen der geisteswissenschaftlichen, die andere Hälfte der naturwissenschaftlichen Studiengänge, jeweils zur Hälfte Frauen und Männer) wurde dreimal (im Abstand von zehn Jahren, beginnend im Jahr 1990) dieselbe offene Frage gestellt: „Podaj, co według Ciebie stanowi o istocie prawdziwej, demo-

\footnotetext{
${ }^{13}$ Siehe Band JWP.
} 
kracji“?“[,Was macht deiner Meinung nach eine echte ,Demokratie’ aus?“] Diese Fragestellung ließ mehrere Antwortmöglichkeiten zu, wobei der darin enthaltene Modifikator „prawdziwy“ nicht zufällig gewählt war, enthielt er doch einen gezielten „modalen Sinn“, der ,in der Umgangssprache zwischen ,ist“ und ,sein soll“ oszilliert“ (Bartmiński, „Zmiany rozumienia“ 15).

Unter Eigenschaften, die das „semantische Zentrum“ des polnischen Demokratiebegriffs mitbestimmten, fanden sich im Laufe der drei Jahrzehnte Freiheit (ASA 1990 - 95 Nennungen, ASA 2000 - 91 Nennungen, ASA 2010 - 95 Nennungen) und Gleichheit (ASA 1990 - 35 Nennungen, ASA 2000 - 33 Nennungen, ASA 2010 - 32 Nennungen). Für die wichtigste Eigenschaft hielten die Befragten dabei die Meinungsfreiheit/Redefreiheit und die Gedankenfreiheit/Freiheit des Denkens, weiter (in dieser Reihenfolge) die Entscheidungsfreiheit und Religionsfreiheit. Unter Gleichheit verstanden die Studierenden hauptsächlich die Gleichheit Aller (Menschen), die Gleichheit der Rechte (und Pflichten), die Gleichheit vor dem Gesetz sowie die Gleichberechtigung. Eine hohe Rangposition erzielten außerdem politische Attribute der Demokratie wie: freie Wahlen, Herrschaft des Volkes/Macht des Volkes, Beteiligung der Bürger an der Staatsgewalt. Im politischen Aspekt seltener genannt wurden Merkmale der Demokratie wie: Rechtsstaatlichkeit, Wahl der Regierung durch freie Wahlen, Rolle jedes Bürgers im Staat /Aufklärung und Engagement der Bürger. Weitere Plätze auf der Rangliste nahmen soziale Merkmale der Demokratie ein wie Toleranz, Respekt für den Einzelnen sowie die Überzeugung, dass die Mehrheit über die Angelegenheiten der Allgemeinheit entscheidet. Als ein wesentliches Attribut von ,Demokratie“ unter ethischen Gesichtspunkten wurde die Gerechtigkeit angesehen (vgl. Majer-Baranowska 72ff.).

Die Art der Vorbereitung und Erstellung der deutschen axiologischen Umfrage war mit dem Vorgehen bei der polnischen „Umfrage zum axiologischen Wörterbuch“ (ASA) identisch. Die Umfrage enthielt die folgende offene Frage, die auf Deutsch gestellt wurde: „Was macht deiner Meinung nach eine echte ,Demokratie“ aus?“. Ziel der Umfrage war es, das ,gängige, allgemeine Wissen“ im Verständnis des Demokratiebegriffs aufzuspüren.

Bei den Eigenschaften, die am deutlichsten hervortraten und die „Kernzone“ der Bedeutung des Ausdrucks ,Demokratie“ in der deutschen Sprache mitgestalteten, positionierten sich - ähnlich wie im Fall des analogen polnischen Ausdrucks - Freiheit (FB 2019 - 76 Nennungen) - insbesondere

\footnotetext{
${ }^{14}$ Die Ergebnisse der Erhebung aus dem Jahre 2010 sollen in einem separaten Band veröffentlicht werden. Die Erhebung für das Jahr 2020 ist noch nicht abgeschlossen.
} 
Redefreiheit/Meinungsfreiheit, darauf folgend Entscheidungsfreiheit und Pressefreiheit - sowie Gleichheit (FB 2019 - 27 Nennungen), wobei Letztere überwiegend als Gleichberechtigung und gleiches Recht/gleiche Rechte für alle aufgefasst wurde. In der politischen Dimension fand sich ganz oben auf der Rangliste die Eigenschaft Mitbestimmung/Recht auf Mitbestimmung, weiter freie Wahlen sowie (aktive) Teilnahme/Partizipation. Als weniger stabile Merkmale wurden verzeichnet: Transparenz, Regierung des Volkes/Macht des Volkes, (Drei)Teilung der Gewalt, Verfassung, Wahlrecht, Politiker als Vertreter des Willens des Volkes und nicht des eigenen Willens. Unter sozialen Gesichtspunkten vervollständigten folgende Attribute das Bild der „echten“ ,Demokratie“: Akzeptanz, Mehrheit entscheidet über Angelegenheiten der Allgemeinheit, Respekt, Toleranz, Meinungsaustausch, Pluralismus, Selbstbestimmung/Unabhängigkeit/Selbständigkeit, (friedliches) Miteinander/Zusammenleben. Unter dem ethischen Aspekt nannten die Befragten außerdem Gerechtigkeit, Ehrlichkeit/aufrechtes Handeln und äußerten die Meinung, dass in der Demokratie jeder Mensch zähle.

Zusammenfassend kann man sagen, dass für junge Deutsche, ähnlich wie für die gleichaltrigen Polen, drei Aspekte des Demokratiebegriffs relevant erschienen, nämlich der soziale, der politische sowie - in geringerem Maße der ethische Aspekt. Andere Aspekte (u.a. der existenzielle oder der kulturelle) wurden für wichtig, jedoch weniger charakteristisch befunden.

\section{DER BASISBEGRIFF DER ,DEMOKRATIE“ IM DEUTSCHEN UND IM POLNISCHEN}

Mithilfe der skizzenhaft herangezogenen Wörterbuch- und Umfragedaten konnte eine Gruppe der sog. „Basiseigenschaften“/Grundeigenschaften erstellt werden, die der ,Demokratie" von Nutzern der deutschen und der polnischen Sprache gleichermaßen zugeschrieben werden und die im Sinne von Teun van Dijk zur „gemeinsamen kulturellen Basis“ („cultural common ground“) gehören.

Etwas verallgemeinert kristallisierten sich in den beiden behandelten Sprachen folgende gemeinsame "Grundelemente“ in der Auffassung des Demokratiebegriffs heraus: [1] die Auffassung von Demokratie - unter dem politischen Aspekt - als einer Form der Herrschaft/Form des politischen Systems, [2] in der das Machtsubjekt (Souverän) das Volk/die Mehrheit der Gesellschaft/alle Bürger sind, [3] von denen jeder über das Recht verfügt, 
mitzuentscheiden sowie über das gleiche Recht, die Form des politischstaatlichen Lebens durch Teilnahme an freien Wahlen zu beeinflussen. In der sozialen Dimension garantiert Demokratie den Menschen/Bürgern [4] umfangreiche Rechte und Freiheiten. Sie garantiert ihnen auch [5] Gleichheit und Gleichberechtigung. Über ihr Wesen bestimmen ferner auch solche Merkmale wie [6] Respektierung des Einzelnen und [7] Toleranz. Unter dem ethischen Aspekt - der im Vergleich zu den beiden vorher genannten Aspekten weniger ausgeprägt ist - ist das Wesen der Demokratie vorrangig durch [8] Gerechtigkeit bestimmt.

\section{DER DEMOKRATIEBEGRIFF IN TEXTEN}

Das Bild des Demokratiebegriffs in den Texten meist überregionaler Tageszeitungen mit hoher Auflage stimmt mit der Auffassung von ,Demokratie' überein, die in den Wörterbüchern erfasst und von den Umfrageergebnissen bestätigt und präzisiert wurde. Aufgrund der immensen Fülle von Texten, die als Grundlage für die Rekonstruktion des Demokratieverständnisses (wie auch vieler anderer Begriffe) herangezogen werden könnten sowie ihrer Vielfalt im Hinblick auf Gattung, Stil und Ideen wurde der Fokus vorrangig auf publizistische, „nicht-klischierte“ Texte gelegt, die „laufend“ entstehen, oft einmalig sind, sich auf „das Kommunizieren einer Neuigkeit“ konzentrieren, dabei jedoch stets ,auf irgendeinem präsupponierten, stereotypen Wissen“ basieren (vgl. Bartmiński und Żuk 52). Diese Texte „richten sich an die sprachlichen Kompetenzen eines Durchschnittspolen [und Durchschnittsdeutschen]. Sie erzeugen ein gemeinsames Werteverständnis und ein gemeinsames Wissen über die Welt." (53).

Um den Rahmen der vorliegenden Arbeit nicht zu sprengen, wurde die Besprechung der Funktionsweise des Demokratiebegriffs in den Texten mit dem Versuch verbunden, seine kontextuelle Varietät zu eruieren und herauszuarbeiten. Orientierung lieferte dabei der Begriff der Profilierung. Der Schwerpunkt lag dabei auf Grundattributen der ,Demokratie', die in verschiedenen Arten des öffentlichen Diskurses unterschiedlichen Modifikationen unterzogen werden, wie etwa: Herrschaft der Mehrheit, Freiheit, Gleichheit, Toleranz, außerdem auch Pluralismus (siehe dazu Fleischer). 


\section{DIE PROFILIERUNG DES DEMOKRATIEBEGRIFFS \\ IN ZWEI AUSGEWÄHLTEN TYPEN DES ÖFFENTLICHEN DISKURSES IN DEUTSCHLAND}

Durch die Analyse des gesammelten Textmaterials und seine Exemplifizierung wurde deutlich, dass dem Basiskonzept der Demokratie auf der Ebene des deutschen öffentlichen Diskurses - unterschiedlich ausgewählte und ausgeprägte - qualitative Merkmale zugeschrieben werden. So entstehen mannigfaltige axiologische und ideologische Varianten des Demokratiebegriffs. In diesem Teil des Beitrags wird die Profilierung des Demokratiebegriffs in Zeitungen und Zeitschriften mit folgenden Ausprägungen skizziert: liberalkonservativ (Frankfurter Allgemeine Zeitung) und linksliberal (Süddeutsche Zeitung, Die Zeit).

Im Diskurs der Medien mit liberalkonservativer Ausrichtung bedeutet Demokratie die Herrschaft der Mehrheit, dabei auch „den Kampf um die Mehrheit" und die Respektierung von Mehrheitsentscheidungen (auch in Weltanschauungsfragen) unter der Annahme, dass die Minderheit zwar Schutz genießt, aber „nicht das Anrecht, die Mehrheit zu überstimmen“. Es werden grundlegende Freiheitsrechte (Grundrechte) geprägt. Freiheit wird mit Verantwortung einerseits sowie mit (innerer und äußerer) Sicherheit andererseits assoziiert. Es wird auf das in der Demokratie zentrale Verfassungsprinzip der Gleichberechtigung von Frauen und Männern verwiesen. Pluralismus wird als Vielfalt von „Ansichten, Bedürfnissen und Interessen“ der Bürger verstanden, wobei implicite u.a. auf den politischen und ideologischen Pluralismus hingewiesen wird.

Aus der Perspektive der linksliberalen Medien setzt die Demokratie die Herrschaft einer (parlamentarischen) Mehrheit voraus, die die Rechte verschiedener Minderheiten respektiert. Demokratie bedeutet die Vereinbarung der Herrschaft der (parlamentarischen) Mehrheit mit Werten wie Respektierung der Rechte von Minderheiten, Führung der öffentlichen Debatten ,auf zivilisierte Art und Weise“, „friedliche Konfliktlösung“ unter Einhaltung demokratischer Prozeduren, „Verzicht auf Gewaltanwendung“. Die Demokratie wird stark mit den in der Verfassung festgehaltenen Grundrechten (Freiheits- und Gleichheitsrechten) verbunden, insbesondere mit verschiedensten Arten von Freiheit. Freiheit steht in engem Zusammenhang mit Verantwortung. Gleichheit bezieht sich in erster Linie auf die von der Verfassung garantierte Gleichberechtigung/Gleichbehandlung von Frauen und Männern. Toleranz und Pluralismus werden als typische, unverzichtbare 
Merkmale einer Demokratie aufgefasst; dabei wird auf den Pluralismus der Medien und den politischen Pluralismus verwiesen. Es wird die Meinung vertreten, dass die Basispolitik in einer Demokratie die Sozialpolitik sei.

\section{DIE PROFILIERUNG DES DEMOKRATIEBEGRIFFS IN ZWEI AUSGEWÄHLTEN TYPEN DES ÖFFENTLICHEN DISKURSES IN POLEN}

Der in der „allgemeinen Auffassung“ verankerte Demokratiebegriff wird in verschiedenen Typen des polnischen öffentlichen Diskurses einer spezifischen Modifizierung (Profilierung) unterzogen. Um eine Vergleichbarkeit mit dem deutschen Diskurs herzustellen, wird nachstehend eine Übersicht über die Profilierung des Demokratiebegriffs in Zeitungen und Zeitschriften mit folgenden Ausrichtungen gegeben: konservativ-liberal (Rzeczpospolita) und linksliberal (Gazeta Wyborcza, Polityka).

Im Diskurs der Medien mit konservativ-liberaler Ausrichtung wird Demokratie mit der Macht/Regierung einer parlamentarischen Mehrheit gleichgesetzt, die Einschränkungen und Kontrolle seitens des Staates unterliegt. Die Übergabe der Macht an die aktuelle Mehrheit führt zur Ablehnung der Ansprüche diverser Minderheitsgruppen. Demokratie wird dabei mit verschiedenen Dimensionen von Freiheit verbunden. In Verbindung gebracht wird sie außerdem mit Gleichheit, aufgefasst als Gleichheit der Bürger vor dem Recht, bzw. als gleiches Recht für alle. Als Grundlage der Demokratie wird auch die Existenz „fester“ und „unveränderbarer" Werte angesehen (insbesondere im Bereich der Sitten), was einen axiologischen und einen Weltanschauungspluralismus ausschließt. Man geht von einem politischen und ideologischen Pluralismus aus, der sich in der Rivalität der politischen Parteien und alternativen Ideen (rechts gegen links, konservativ gegen liberal) bzw. im Aufeinanderprallen diverser Vorstellungen von dem, was (politisch und ideologisch) wahr und richtig ist, offenbart.

Im Diskurs der linksliberalen Medien geht Demokratie von der Herrschaft einer (parlamentarischen) Mehrheit aus, die die Rechte diverser Minderheiten respektiert. Sie geht von der Freiheit aus, die in die Prinzipien des Rechtsstaates eingefügt und mit Verantwortung fest verbunden ist. Es wird betont, dass Freiheit und Autonomie jedem Einzelnen „vollständig“ zustehen. Gleichheit bedeutet gleiche Rechte und Chancen, die in der Praxis jeder „auf eigene Rechnung“ nutzen muss (es wird konstatiert, dass im 
Kampf um Erfolg „die Besseren gewinnen“). Mit dem Demokratiebegriff verbindet man einen breit aufgefassten Pluralismus (axiologisch, politisch, Pluralismus der Weltanschauung und der Kultur), der eine Vielfalt gleichwertiger Moral- und Wertesysteme (darunter der liberalen Werte), Glaubensrichtungen und Religionen, Überzeugungen, Ansichten, Gesinnungen, Lebensstile und Lebensphilosophien, Wahrheiten, Meinungen und Interessen verschiedener sozialer und politischer Gruppen sowie eine breite Anzahl an politischen Vorstellungen voraussetzt. Begleitet wird er von der Toleranz, aufgefasst als Respektierung von Anderssein und Diversität, Anerkennung, Achtung und Schutz verschiedener Minderheiten, ihrer Rechte und Interessen.

\section{ZUSAMMENFASSUNG}

Zusammenfassend lässt sich sagen, dass die „Kernbedeutung“ des Demokratiebegriffs in beiden Sprachen weitgehend übereinstimmt. Auf ihn bezieht sich - mehr oder weniger - jede Art des deutschen und des polnischen öffentlichen Diskurses, der dabei einen Standpunkt widerspiegelt, der mit der angenommenen Weltanschauung und dem berücksichtigten (akzeptierten) Wertesystem übereinstimmt. Im polnischen öffentlichen Diskurs wird dabei eine größere Differenzierung der der ,Demokratie' zugewiesenen Eigenschaften deutlich, während der deutsche öffentliche Diskurs in dieser Hinsicht ein einheitlicheres Verständnis offenbart.

Bezeichnend ist bei allen Arten des deutschen öffentlichen Diskurses der Bezug auf den Katalog der sog. Grundrechte (insbesondere der Freiheitsrechte), die von der Verfassung garantiert sind und die für einen unbestreitbaren Wert gehalten werden; außerdem der Bezug auf das konstitutionelle Prinzip der Gleichheit zwischen Frauen und Männern. Charakteristisch ist zudem die Auffassung von ,Demokratie“ in der ursprünglichen Bedeutung dieses Begriffs. Pluralismus wird in einer Demokratie als selbstverständlich angesehen. Dabei werden dessen unterschiedliche Ausprägungen betont, wie etwa der politische Pluralismus (liberalkonservativer und linksliberaler Diskurs) und der mediale Pluralismus (linksliberaler Diskurs). Betont wird die interkulturelle Toleranz, deren Wesen in der Akzeptanz von Menschen anderer Kulturen und Nationalitäten Ausdruck finden soll.

Im Rahmen des polnischen öffentlichen Diskurses lassen sich zwei entgegengesetzte Pole beobachten. Der linksliberale Diskurs korrespondiert mit dem hier aus Platzgründen nicht besprochenen linken Diskurs in Fragen be- 
züglich des Weltanschauungs- und axiologischen Pluralismus, des Schutzes der Rechte (verschiedener) Minderheiten, die eigene Meinung zu vertreten und die eigene Weltanschauung zu äußern. Auf der anderen Seite positionieren sich Medien mit konservativ-liberaler Ausrichtung, in denen die Annahme der Existenz fester Werte in der Demokratie den axiologischen und den Weltanschauungspluralismus ausschließt.

Unterschiede in der Profilierung des Demokratiebegriffs im deutschen und polnischen öffentlichen Diskurs äußern sich in zwei Grunddimensionen, nämlich: a. auf Ebene der einzelnen Diskurse der jeweiligen Nationalsprache (Deutsch, Polnisch); b. in der deutsch-polnischen Vergleichsperspektive im Bereich der sich entsprechenden Diskurstypen.

\section{BIBLIOGRAFIE}

VERZEICHNIS DER DEUTSCHEN WÖRTERBÜCHER:

AFW Hey - Heyse, Johann Christian August. Allgemeines verdeutschendes und erklärendes Fremdwörterbuch mit Bezeichnung der Aussprache und Betonung der Wörter nebst genauer Angabe ihrer Abstammung und Bildung. Neu bearb. von Karl Wilhelm Ludwig Heyse. Ausgabe 10, Hahnsche Hof-Buchhandlung, [1804]/1848.

DEW Köb - Köbler, Gerhard. Deutsches Etymologisches Wörterbuch, 1995; http://www.koebler gerhard.de/derwbhin.html. Abruf am 30.06.2020.

DudBW - Duden. Bedeutungswörterbuch, hrsg. und bearb. von Wolfgang Müller. Aufl. 2 (völlig neu bearbeitet und erweitert), Bd. 10, Dudenverlag, 1985.

DudDUW - Duden. Deutsches Universalwörterbuch, bearb. von Günther Drosdowski und der Dudenredaktion. Aufl. 3 (neu bearbeitet und erweitert), Dudenverlag, [1983]/1996.

DudEHW - Duden. Etymologie. Herkunftswörterbuch der deutschen Sprache, bearb. von Günther Drosdowski, Bd. 7. Aufl. 2, Dudenverlag, 1989.

DudFW - Duden. Fremdwörterbuch, hrsg. und bearb. vom Wissenschaftlichen Rat der Dudenredaktion, redaktionelle Bearbeitung Werner Scholzestubenrecht. Ausgabe 6 (auf der Grundlage der amtlichen Neuregelung der deutschen Rechtschreibung überarbeitete und erweiterte Ausgabe), Bibliographisches Institut \& F.A. Brockhaus AG, 1997.

DudSW - Duden. Stilwörterbuch der deutschen Sprache. Die Verwendung der Wörter im Satz, völlig neu bearbeitet und erweitert von Günther Drosdowski, Bd. 2. Aufl. 7, Dudenverlag, 1988.

DW Mack - Mackensen, Lutz. Deutsches Wörterbuch, Bd. 1-3, Rowohlt Taschenbuch Verlag $\mathrm{GmbH}, 1979$.

DW Paul - Paul, Hermann. Deutsches Wörterbuch. Bedeutungsgeschichte und Aufbau unseres Wortschatzes. Aufl. 10 (überarbeitete und erweiterte von Helmut Henne u.a.), Niemeyer, [1897]/2002.

DW Wah - Wahrig, Gerhard. Deutsches Wörterbuch, neu hrsg. von Renate Wahrig-Burfeind, Bertelsmann Lexikon Verlag, 1997. 
EW Klu - Kluge, Friedrich. Etymologisches Wörterbuch der deutschen Sprache, bearb. von Elmar Seebold. Aufl. 24 (durchgesehen und erweitert), Walter de Gruyter, [1883]/2001.

FWB - Frühneuhochdeutsches Wörterbuch, hrsg. von Ulrich Goebel u.a., begründet von Robert R. Anderson u.a., Bd. 1-11, Walter de Gruyter, 1989-2013 [Bd. 5, Lieferung 1, d-deube, 2006, bearb. von Markus Denkler u.a.].

EWD Pfei - Pfeifer, Wolfgang. Etymologisches Wörterbuch des Deutschen, erarbeitet im Zentralinstitut für Sprachwissenschaft. Aufl. 2, Akademie-Verlag, 1993 [Deutscher Taschenbuch Verlag, 1995].

FW San - Sanders, Daniel. Fremdwörterbuch, Bd. 1-2, Verlag von Otto Wigand, 1871.

GKW Adel - Grammatisch-kritisches Wörterbuch der hochdeutschen Mundart mit beständiger Vergleichung der übrigen Mundarten, besonders aber der oberdeutschen, hrsg. von Johann Christoph Adelung u.a., Bd. 1-4, Bauer, 1811 [Aufl. 1, 1774-1786, 5 Bde.].

GW Mül - Müller, Wolfgang. Das Gegenwort-Wörterbuch. Ein Kontrastwörterbuch mit Gebrauchshinweisen. Walter de Gruyter, 1998.

HW Kemp - Handwörterbuch der deutschen Gegenwartssprache, von einem Autorenkollektiv unter der Leitung von Günter Kempcke, Bd. 1-2, Akademie-Verlag, 1984.

REW - Mackensen, Lutz. Reclams Etymologisches Wörterbuch der deutschen Sprache. Philipp Reclam jun., 1966.

WDG KlapStein - Wörterbuch der deutschen Gegenwartssprache, hrsg. von Ruth Klappenbach und Wolfgang Steinitz, Bd. 1-6, Akademie-Verlag, 1964-1977.

VERZEICHNIS DER POLNISCHEN WÖRTERBÜCHER

ISJP Bań - Inny słownik języka polskiego, hrsg. von Mirosław Bańko, Bd. 1-2, Wydawnictwo Naukowe PWN, 2000.

ISJP Sob - Ilustrowany słownik języka polskiego, hrsg. von Elżbieta Sobol, Wydawnictwo Naukowe PWN, 1999.

NSEJP Dług-Kur - Długosz-Kurczabowa, Krystyna. Nowy słownik etymologiczny języka polskiego. Wydawnictwo Naukowe PWN, 2003.

PSWP Zgół - Praktyczny słownik współczesnej polszczyzny, hrsg. von Halina Zgółkowa, Bd. 1-50, Wydawnictwo Kurpisz, 1994-2005.

SIJP - Arct, Michał. Stownik ilustrowany języka polskiego. Aufl. 3, [1916]/1929.

SJP Dor - Słownik języka polskiego, hrsg. von Witold Doroszewski, Bd. 1-11, Wydawnictwo Naukowe PWN, 1958-1969.

SJP DSSW - Stownik języka polskiego PWN, hrsg. von Lidia Drabik u.a., Wydawnictwo Naukowe PWN, 2006.

SJP Szym - Stownik języka polskiego, hrsg. von Mieczysław Szymczak, Bd. 1-3, Wydawnictwo Naukowe PWN, 1978-1981 [zahlreiche Nachdrucke, Supplementband 1992].

SL - Linde, Samuel Bogumił. Stownik języka polskiego, Bd. 1-6, 1807-1814 [Aufl. 2, 18541860].

SPXVI - Słownik polszczyzny XVI wieku, red. Maria Renata Mayenowa, Franciszek Pepłowski, Bd. 1-34; red. Krzysztof Mrowcewicz, Patrycja Potoniec, Bd. 35; red. nacz. Krzysztof Mrowcewicz, zastępca red. nacz. Patrycja Potoniec, kom. red. Jerzy Bartmiński, Bd. 36; red. nauk. Patrycja Potoniec, Bd. 37; Ossolineum, 1966-1994; IBL PAN, 1995, 2012, 2019.

SSiA - Pawlus, Marta, und Beata Gajewska, Stownik synonimów i antonimów. PPU „Park” Sp. Z o.o., 1999. 
SSP Kurz - Stownik synonimów polskich, hrsg. von Zofia Kurzowa, Wydawnictwo Naukowe PWN, 2001.

SSP Kras - Krasiński, Adam Stanisław, Stownik synonimów polskich, Bd. 1-2, Drukarnia „Czasu” Fr. Kluczyckiego i sp., 1885.

SW - Karłowicz, Jan, u. Adam Kryński, Władysław Niedźwiedzki, Słownik języka polskiego, Bd. 1-8, 1900-1927 [wyd. fotooffsetowe: PIW, 1952-1953].

SWB LPT-H - Słownik wyrazów bliskoznacznych, hrsg. von Danuta Ludwiczak u.a., Wiedza Powszechna, 1998.

SWil - Zdanowicz, Aleksander u.a., Stownik języka polskiego, Bd. 1-2, Wyd. Maurycego Orgelbranda, 1861.

SWJP Dun - Słownik współczesnego języka polskiego, hrsg. von Bogusław Dunaj, Bd. 1-5, Wydawnictwo SMS, 2000-2003 [Aufl. 1, Wydawnictwo Wilga, 1996].

USJP Dub - Uniwersalny słownik języka polskiego PWN, hrsg. von Stanisław Dubisz, Bd. 1-4, Wydawnictwo Naukowe PWN, 2003.

ACE - Bartmiński, Jerzy. Aspects of Cognitive Ethnolinguistics. Equinox, 2009.

Bartmiński, Jerzy. „Czym zajmuje się etnolingwistyka.” Akcent, Nr 4, 1986, S. 16-22.

Bartmiński, Jerzy. „Pasywne i aktywne paneuropeizmy we współczesnym języku polskim.” Słownictwo wspótczesnej polszczyzny w okresie przemian, hrsg. von Jan Mazur, Wydawnictwo UMCS, 2000, S. 109-116.

Bartmiński, Jerzy. „Zmiany rozumienia nazw wartości w okresie transformacji ustrojowej w Polsce.” Język - wartości - polityka. Zmiany rozumienia nazw wartości w okresie transformacji ustrojowej $w$ Polsce. Raport z badań empirycznych, hrsg. von Jerzy Bartmiński, Wydawnictwo UMCS, 2006, S. 8-35.

Bartmiński, Jerzy. „Wartości i ich profile medialne.” Oblicza komunikacji. Ideologie w stowach i obrazach, hrsg. von Irena Kamińska-Szmaj, Tomasz Piekot, Marcin Poprawa, Wydawnictwo Uniwersytetu Wrocławskiego, 2008, S. 23-41.

Bartmiński, Jerzy, und Stanisława Niebrzegowska. „Profile a podmiotowa interpretacja świata.” Profilowanie $w$ języku $i w$ tekście, hrsg. von Jerzy Bartmiński, Ryszard Tokarski, Wydawnictwo UMCS, 1998, S. 211-224.

Bartmiński, Jerzy, und Stanisława Niebrzegowska-Bartmińska. Tekstologia. Wydawnictwo Naukowe PWN, 2009.

Bartmiński, Jerzy, und Grzegorz Żuk. „Pojęcie RÓWNOŚCI i jego profilowanie we współczesnym języku polskim." Etnolingwistyka, Bd. 21, 2009, S. 47-67.

Dijk, Teun A. van. „Dyskurs polityczny i ideologia.” Übersetzt von Aneta Wysocka. Etnolingwistyka, Bd. 15, 2003, S. 7-28.

Fleischer, Michael. „Symbolika kolektywna w Polsce i w Niemczech (porównanie interkulturowe)." Etnolingwistyka, Bd. 11, 1999, S. 69-106.

Grzeszczak, Monika. „Der Begriff demokracja ,Demokratie’ im Gegenwartspolnisch. Versuch einer kognitiven Definition.“ Europa und seine Werte. Akten der internationalen Arbeitstagung „Normen und Wertbegriffe in der Verständigung zwischen Ost- und Westeuropa" 3./4. April 2008 in Lublin, Polen, hrsg. von Jerzy Bartmiński, Rosemarie Lühr, Peter Lang, 2009, S. 41-55. 
Grzeszczak, Monika. Pojęcie DEMOKRACJI i jego profilowanie w polskim i niemieckim dyskursie publicznym (1989-2009). Towarzystwo Naukowe KUL, 2015.

Grzeszczak, Monika. „Stabilität und Variabilität des Demokratiebegriffs im Deutschen.“ Werte und Paradigmen zwischen Wandel und Kontinuität. Literatur- und sprachwissenschaftliche Perspektiven, hrsg. von Anna Rutka, Magdalena Szulc-Brzozowska, Vandenhoeck@ Ruprecht Verlage, 2019a, S. 221-238.

Grzeszczak, Monika. „Pojęcie DEMOKRACJI w języku polskim.” Wartości w językowo-kulturowym obrazie świata Stowian i ich sąsiadów 5, hrsg. von Stanisława Niebrzegowska-Bartmińska, Dorota Pazio-Wlazłowska, Wydawnictwo UMCS, 2019b, S. 201-222.

JOS - Językowy obraz świata, hrsg. von Jerzy Bartmiński. Wydawnictwo UMCS, 1990 [zahlreiche Neuveröffentlichungen].

JWP - Język - wartości - polityka. Zmiany rozumienia nazw wartości w okresie transformacji ustrojowej $w$ Polsce. Raport z badań empirycznych, hrsg. von Jerzy Bartmiński. Wydawnictwo UMCS, 2006.

Majer-Baranowska, Urszula. „DEMOKRACJA.” Język - wartości - polityka. Zmiany rozumienia nazw wartości w okresie transformacji ustrojowej w Polsce. Raport z badań empirycznych, hrsg. von Jerzy Bartmiński, Wydawnictwo UMCS, 2006, S. 72-77.

Niebrzegowska-Bartmińska, Stanisława, „Badania etnolingwistyczne w Lublinie.” Poznańskie Spotkania Językoznawcze, hrsg. von Zdzisława Krążyńska, Zygmunt Zagórski, Bd. 13, 2004, S. 79-89.

PJT - Profilowanie w języku $i$ w tekście, hrsg. von Jerzy Bartmiński, Ryszard Tokarski. Wydawnictwo UMCS, 1998.

PP - Profilowanie pojęć. Wybór prac, hrsg. von Jerzy Bartmiński. Wydawnictwo UMCS, 1993.

Waszakowa, Krystyna. Przejawy internacjonalizacji w słowotwórstwie współczesnej polszczyzny. Wydawnictwo Uniwersytetu Warszawskiego, 2005.

\section{POJĘCIE DEMOKRACJI \\ I JEGO PROFILOWANIE W JĘZYKU NIEMIECKIM I POLSKIM. ANALIZA KONTRASTYWNA}

\section{Streszczenie}

Celem artykułu jest analiza kontrastywna rozumienia pojęcia demokracji w języku niemieckim i polskim oraz pokazanie sposobów jego konceptualizacji we współczesnym niemieckim i polskim dyskursie publicznym. Podstawę metodologiczną stanowią założenia lubelskiej etnolingwistyki kognitywnej. Materiał językowy został zaczerpnięty z trzech typów źródeł: ze słowników języka niemieckiego i polskiego, z empirycznych badań ankietowych oraz z tekstów prasowych. Na jego podstawie ustalono zespół podstawowych cech definicyjnych w rozumieniu 'demokracji' (tzw. zespół „cech bazowych”) wspólny różnym dyskursom w obu odnośnych językach. W kolejności wskazano zespoły cech przypisywanych 'demokracji' na gruncie wyspecjalizowanych dyskursów zróżnicowanych ideologicznie i światopoglądowo. Profilowanie pojęcia 'demokracji' zostało zarysowane na podstawie materiału tekstowowego zaczerpniętego z prasy niemieckiej i polskiej o orientacji liberalno-konserwatywnej i lewicowo-liberalnej.

Słowa kluczowe: demokracja; dyskurs; profilowanie; słownik; ankieta. 


\section{THE CONCEPT OF DEMOCRACY \\ AND ITS PROFILING IN GERMAN AND POLISH: \\ A CONTRASTIVE ANALYSIS}

\section{S u m m a ry}

The purpose of this paper is a contrastive analysis of the concept of 'democracy' in the German and Polish languages, along with a presentation of the modes of its conceptualisation in modern Polish and German public discourse. The methodological basis is provided by the assumptions of the Lublin school of cognitive ethnolinguistics. The linguistic materials derive from three types of sources: dictionaries of the German and Polish languages, empirical surveys and press texts. Based on these, a set of basic definition features were defined in the understanding of 'democracy' (the so-called "set of basic features") common for various discourses in both languages. The sets of features assigned to 'democracy' on the basis of specialist discourses, diverse both ideologically and with respect to their worldview, were listed. The profiling of the concept of 'democracy' was outlined on the basis of texts derived from both the German and Polish press with liberal-conservative and leftist-liberal profiles.

Keywords: democracy; discourse; profiling; dictionary; survey. 